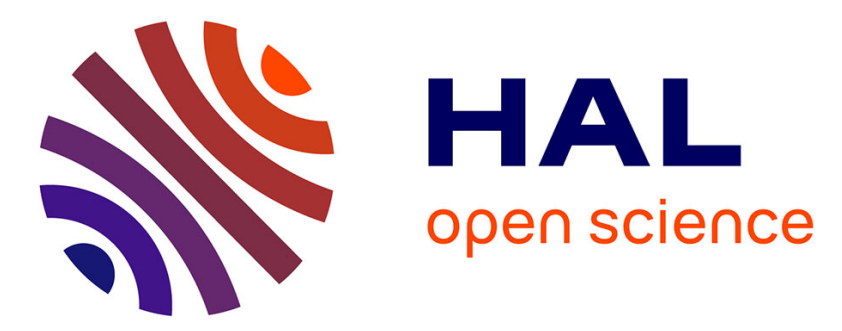

\title{
Galactic cosmic-ray energy spectra and expected solar events at the time of future space missions
}

\author{
C Grimani, H M Araújo, M Fabi, A Lobo, I Mateos, D N A Shaul, T J
}

Sumner, P Wass

\section{- To cite this version:}

C Grimani, H M Araújo, M Fabi, A Lobo, I Mateos, et al.. Galactic cosmic-ray energy spectra and expected solar events at the time of future space missions. Classical and Quantum Gravity, 2011, 28 (9), pp.94005. 10.1088/0264-9381/28/9/094005 . hal-00694879

\section{HAL Id: hal-00694879 \\ https://hal.science/hal-00694879}

Submitted on 7 May 2012

HAL is a multi-disciplinary open access archive for the deposit and dissemination of scientific research documents, whether they are published or not. The documents may come from teaching and research institutions in France or abroad, or from public or private research centers.
L'archive ouverte pluridisciplinaire HAL, est destinée au dépôt et à la diffusion de documents scientifiques de niveau recherche, publiés ou non, émanant des établissements d'enseignement et de recherche français ou étrangers, des laboratoires publics ou privés. 


\title{
Galactic cosmic-ray energy spectra and expected solar events at the time of future space missions
}

\author{
C. Grimani ${ }^{1,2}$, H.M. Araújo ${ }^{3}$, M. Fabi ${ }^{1}$, \\ A. Lobo ${ }^{4}$, I. Mateos ${ }^{4}$, \\ D.N.A. Shaul ${ }^{3}$, T.J. Sumner ${ }^{3}$, P. Wass $^{3}$ \\ ${ }^{1}$ Dipartimento M F I, Università degli Studi di Urbino "Carlo Bo", Urbino (PU) \\ ${ }^{2}$ INFN, Florence, Italy \\ ${ }^{3}$ Imperial College, London, UK \\ ${ }^{4}$ Institut d'Estudis Espacials de Catalunya (IEEC), Barcelona, Spain \\ catia.grimani@uniurb.it
}

\begin{abstract}
Galactic cosmic-rays (GCRs) and solar energetic particles (SEPs) affect observations on board long-lived space missions. We developed a parameterization of proton and helium fluxes for various levels of solar modulation during opposite polarity periods. In addition to long-term variations (decades), short-term fluctuations (minutes to days) were considered as well. In particular, we focused on data from experiments carrying magnetic spectrometers in space. The shortest GCR variations we were able to study are of the order of hours. We point out that GCR variations and fluctuations are strongly energy dependent. Energy dependent is also the detector charging on board space experiments. The measurements of energy differential fluxes and their variations are needed in order to evaluate properly the performance of future space missions. We present here the projections for the GCR fluxes and solar events at the time of LISA (Laser Interferometer Space Antenna) Pathfinder (LISA-PF).

Keywords: Cosmic rays. Sun: solar-terrestrial relations. Space interferometers.
\end{abstract}


PACS number: 95.55Ym, 04.80.Nn, 96.50.sb, 96.50.Vg

\section{Introduction}

Solar activity level, drift of opposite charge particles in the global solar magnetic field (GSMF) and interplanetary processes affect cosmic-ray observations in the local interplanetary medium. Experiments devoted to cosmic-ray physics aim to infer from near-Earth measurements the interstellar spectra. In all other missions energetic particles might affect the performance of the onboard detectors. In LISA-PF and LISA, for example, solar and galactic proton and helium particles above $100 \mathrm{MeV}$ per nucleon $(\mathrm{MeV} / n)$ limit the mission performance charging the onboard test masses (see for details [1]-[2]). In particular, short-term GCR fluctuations generate spurious signals in the experiment band [3] and SEPs associated with strong solar events overcome the whole mission noise budget in the low frequency range (see for example $[4])$.

Predictions of future solar cycle amplitudes allow us to estimate the intensity of GCR and the number of expected solar events during the next decades. The sunspot number is the most widely used proxy for solar activity prediction. In this work we adopt the projections of the next solar cycle intensity based on the observed trend of the solar spot number during the first months of this year [5]-[6]. According to these predictions, we estimate the GCR energy spectra and the number of solar events at the time of LISA Pathfinder at the end of 2012. The number of solar events were estimated according to the Nymmik's model [7]-[8]. Details of the LISA-PF mission are reported in [9].

Part of this work was carried out earlier, however an update was needed since the actual trend shown by the initial rise of the solar cycle 24 appears weaker than projections available up to 2008 [10].

Various theoretical models were proposed in the literature to take into account the effects of solar modulation and solar polarity on GCRs (see for example [11]-[12]). At this time we prefer an empirical approach based on data gathered by experiments carrying magnetic spectrometers in space that must be used to calibrate theoretical models. Proton, helium and electron data were considered. The method was discussed accurately in [13]. Our predictions will be redundantly tested by cosmic-ray experiments in flight at the time of LISA-Pathfinder such as AMS [14] and by the onboard monitors 
of incident solar and galactic proton and helium nuclei above a few tens of $\mathrm{MeV} / \mathrm{n}[15]$.

GCR short-term fluctuations are discussed here in addition to long-term variations. Among short-term fluctuations we include the 27-day variations related to the Sun rotation, Forbush decreases (see Section 3.2 below) and variations of the order of hours. Cosmic-ray fluctuations could not be studied in smaller intervals of time. This limitation arises from the large statistical uncertainties affecting differential flux measurements carried out by experiments with small geometrical factors resulting from the use of magnetic spectrometers.

For completeness we add that studies of GCR fluctuations down to minutes were carried out, for example, by Starodubtsev, Usoskin and Mursala [16] using ground neutron monitors.

\section{Solar cycle 24 projections and observed ini- tial rise}

Predictions of a solar cycle include both amplitude and timing. Timing depends on the characteristics of the solar minimum. Due to the unusual long duration of the last solar minimum we presently expect the next maximum to occur in 2013. Present projections for the solar cycle 24 are reported in the right panel of fig.1 [5]-[6]. Solar cycle 24 projections available up to 2008 according to Hathaway and Dikpati [10] appear in the left panel of fig.1. An extensive review of the solar cycle 24 projections is reported in [17]. Unfortunately, the majority of these predictions were very different from the actual trend shown by the first phase of the solar cycle 24 . In particular, out of 54 predictions, 4 only reported a solar maximum to occur beyond 2012. Intensities ranging between 185 and less than 40 for the average annual sunspot number $\left(R_{24}\right)$ were proposed. However, $78 \%$ of the whole sample of predictions indicated $R_{24}$ above 100 at the maximum. In other words, almost the totality of predictions presented a solar cycle of mediumstrong amplitude. Conversely, the anomalous long duration of the last solar minimum and the trend of the rise of the solar cycle 24 indicate approximately $R_{24}=77 \pm 20$. We point out that a quite good projection was reported by Kontor [18] for both $R_{24}(70 \pm 17.5)$ and timing (2012.96). Moreover, Li, Gao and $\mathrm{Su}[19]$ indicated an average annual sunspot number of 80 and the 

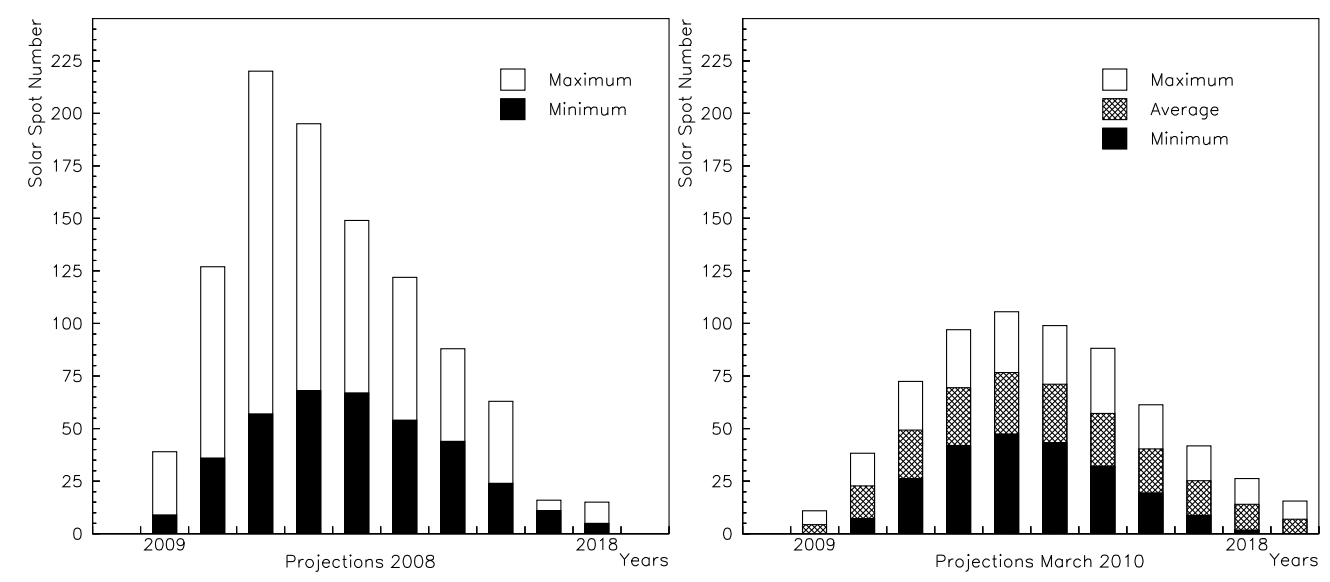

Figure 1: Predictions of the average annual sunspot number for the solar cycle 24: 2008 [10] and updated [5]-[6] projections are compared.

maximum to be reached in February 2013 ( \pm 8 months) in case of a slow riser cycle.

\section{Galactic cosmic-ray proton and helium en- ergy spectra at the time of LISA-PF}

Assuming that LISA-PF will be launched during the first quarter of 2012 and that about 3 months will be needed to reach its final orbit in L1, data will be taken during the second half of 2012, near the next solar maximum and possibly during the same negative polarity epoch we are presently experiencing. We recall that the GSMF polarity is positive (negative) when solar magnetic field lines are directed outward (inward) from the Sun northern pole. Galactic cosmic-ray observations near Earth are affected by both solar activity level and solar polarity [20]. The effect of the solar modulation on GCRs during positive polarity periods is well represented by the symmetric model in the force field approximation by Gleeson and Axford [21]. This model (see eq.(1)) allows us to estimate through an energy loss parameter, $\Phi$, the fluxes of cosmic rays at a distance $r$ from the Sun, at the time $t(J(r, E, t))$ assuming time-independent interstellar fluxes $(J(\infty, E+\Phi))$. In eq.(1) $E$ 
Table 1: Proton (p) and helium (He) flux parameterization at the interstellar medium.

\begin{tabular}{l|ccc}
\hline & A & P1 & P2 \\
\hline $\mathrm{p}$ & $(1.94 \pm 0.13) \times 10^{4}$ & $0.70 \pm 0.52$ & $2.76 \pm 0.03$ \\
$\mathrm{He}$ & $(7.10 \pm 0.56) \times 10^{3}$ & $0.50 \pm 0.31$ & $2.78 \pm 0.03$ \\
\hline
\end{tabular}

is the particle total energy and $E_{o}$ the rest mass. For protons and helium nuclei above rigidities (particle momentum per unit charge [22]) of $100 \mathrm{MV}$ a modulation potential $\phi$, given in units of $M V$, is such that $\Phi=|Z|$ e $\phi$ corresponds to the average energy loss from the interstellar medium to a distance $r$ from the Sun.

$$
\frac{J(r, E, t)}{E^{2}-E_{0}^{2}}=\frac{J(\infty, E+\Phi)}{(E+\Phi)^{2}-E_{0}^{2}}
$$

The modulation potential can be correlated with both neutron monitor countrate [23] and solar spot number [24]. At the moment, the expected number of minimum, average and maximum average annual solar spots in 2012 are 42.03, 69.53 and 97.03, respectively. Very similar to 2003 (40.18, $67.18,94.18$ respectively; [6]). The estimated solar modulation parameter in 2003 was $959 \mathrm{MV}$ [25]. Moreover, the proton data trend observed by the BESS (Balloon-borne Experiment with a Superconducting Spectrometer [26]) experiment in 2002 and 2004 indicated a solar modulation parameter for protons of $1109 M V$ and $764 M V$, respectively (see fig.2). On the basis of these two last evidences we set to $950 \mathrm{MV}$ the solar modulation parameter as a lower limit for the second half of 2012 .

We use the model by Gleeson and Axford to estimate the particle energy spectra during a positive polarity epoch (continuous lines in figs. 2 and 3 for protons and helium nuclei, respectively. References to data are reported in [13]).

Flux interpolation at the interstellar medium was gathered from [27] and reported in eq. (2). The parameters for proton and helium fluxes appear in Table 1 . With $\beta$ and $R$ we indicate particle velocity and rigidity, respectively.

$$
J(\infty, \beta, R)=A \beta^{P 1} R^{-P 2}
$$


Table 2: Proton and helium flux parameterization at $1 A U$ at the time of LISA-PF assuming a positive solar polarity.

\begin{tabular}{l|cccc}
\hline Particle fluxes & $\mathrm{A}$ & $\mathrm{B}$ & $\alpha$ & $\gamma$ \\
\hline $\mathrm{p}$ & 18000 & 1.50 & 3.90 & 1.10 \\
$\mathrm{He}$ & 850 & 1.30 & 3.23 & 0.48 \\
\hline
\end{tabular}

The energy spectra during a negative polarity epoch were estimated according to [13] for near-solar-maximum conditions (dashed lines in figs.2 and 3). Presently, the Sun has a negative polarity. As pointed out in the previous Section, a change of polarity from - to + , is plausibly expected in 2013. Since at solar maximum a minor effect of solar polarity is observed on cosmic rays [28], we consider our estimated fluxes a lower limit at the time of LISA-PF.

The particle differential flux interpolation function at $1 A U$ we use is [29]:

$$
F(E)=A(E+B)^{-\alpha} E^{\gamma} \text { Particles } /\left(m^{2} \text { srsGeV }\right) .
$$

Eq. 3 parameters for proton and helium nucleus flux interpolation are reported in Table 2.

\section{Galactic cosmic-ray short-term variations and fluctuations}

\section{$3.1 \quad 27$-day variations}

The Sun is a massive sphere of plasma and gas rotating at different rates depending on the heliolatitudes. The equator and near equatorial regions of the Sun rotate with a period of about 25-26 days: the Sun's sideral rotation period. For an observer at the Earth this periodicity equals about 27-28 days due to the orbital motion of the Earth. This is called the Sun's sinodic period of rotation. At the poles the Sun rotates with a period of about 36 days. The distribution of active regions and coronal holes on the Sun generates a solar wind asymmetric velocity distribution with respect to heliolongitude and heliolatitude. Since the Sun rotation depends on the reference system of the observer, these recurrent variations are called 27-day variations of GCR intensity [30]. 


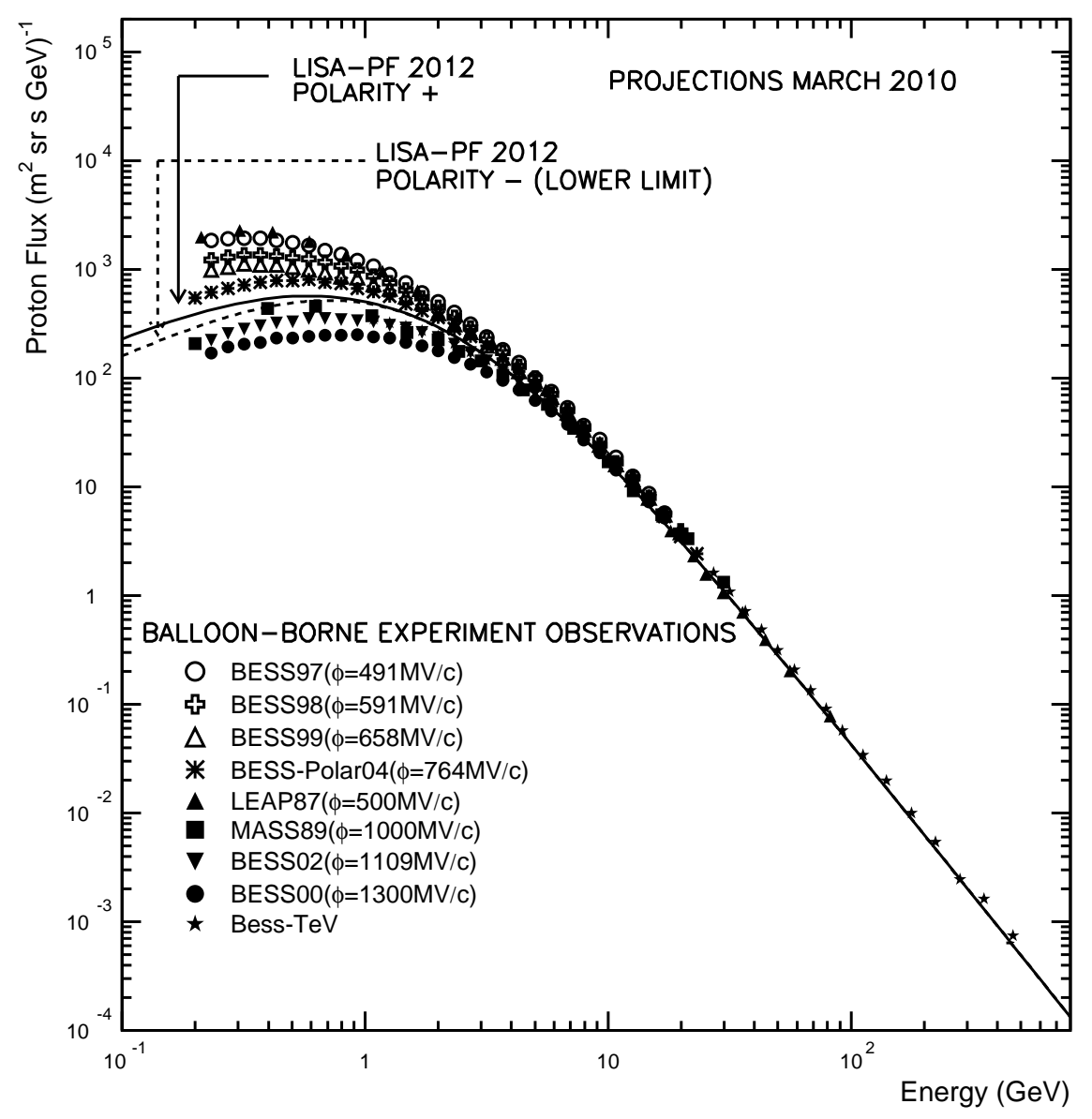

Figure 2: Estimated GCR proton energy spectra at the time of LISA-PF (2012). 


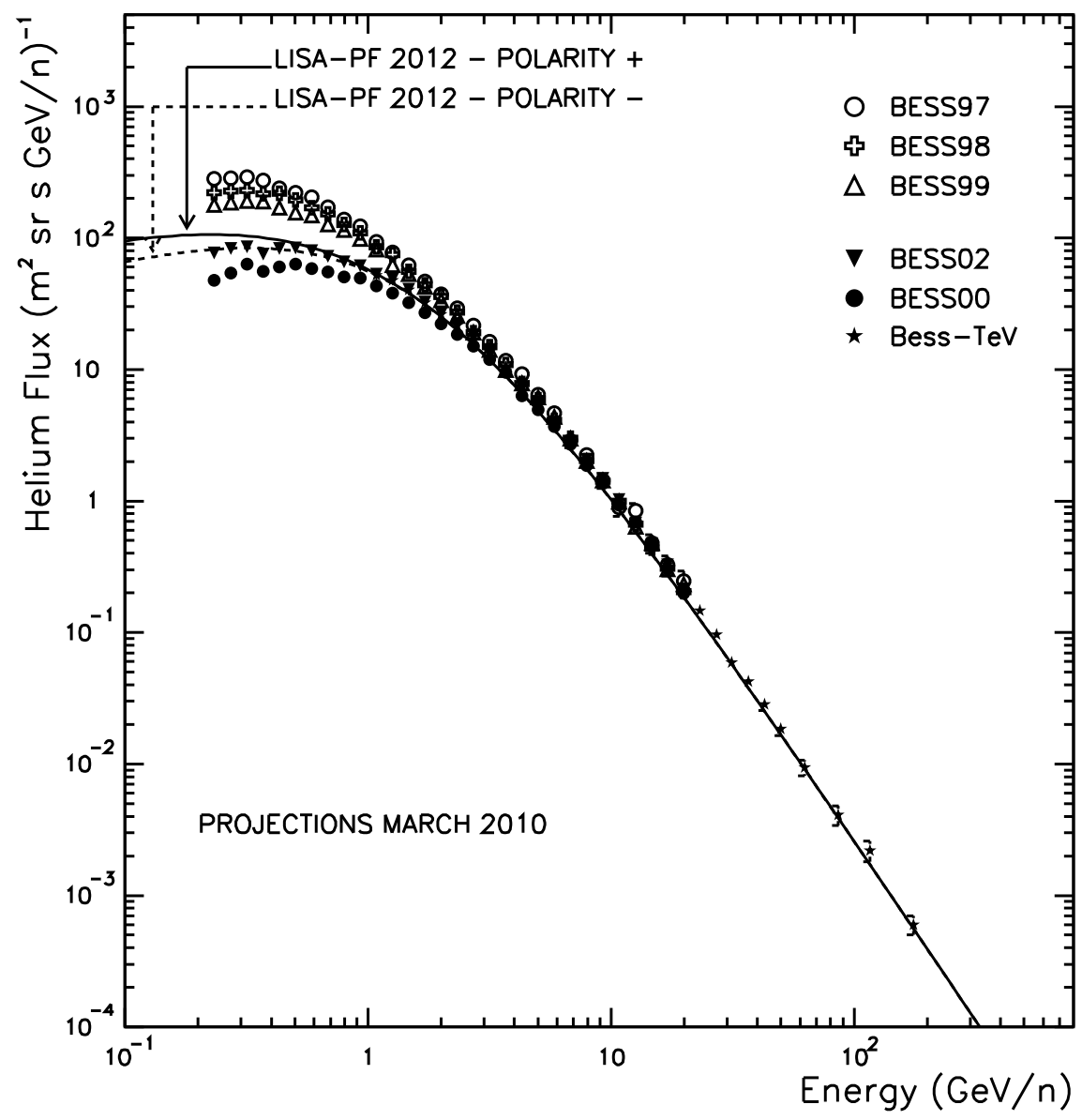

Figure 3: Same as fig.2 for helium nuclei. 
Alania, Gil and Modzelewska [31] showed that the larger amplitudes of the 27-day variations of the GCR intensity and anisotropy are observed during periods of minimum solar activity and positive polarity. In particular, they found that the amplitudes of the 27-day intensity variations present a power-law spectrum in rigidity $\left(A_{27}=\mathrm{b} R^{-\delta}\right)$. This spectrum appears hard $(\delta=0.54 \pm 0.11)$ during positive polarity periods and soft $(\delta=0.95 \pm 0.12)$ during negative polarity epochs. From the data reported by Alania, Gil and Modzelewska we have inferred that $A_{27}$ varies between $6 \%$ at $0.445 G V$ (0.1 $\mathrm{GeV}$ for protons) and $1.2 \%$ at $10 \mathrm{GV}$ at solar minimum during positive polarity epochs while goes down to $4 \%$ at $0.445 G V$ and $0.2 \%$ at $10 G V$ during negative polarity periods. GCR anisotropy is smaller than $0.06 \%(0.03 \%)$ during positive (negative) polarity periods.

The 27-day variations were not found to depend on the tilt angles of the heliospheric current sheets according to neutron monitor data.

\subsection{Forbush decreases}

Forbush decreases were discovered by Forbush in 1937. A Forbush decrease is a worldwide drop of the observed GCR intensity occurring within tens of minutes to hours followed by a gradual recovery to the previous average intensity within many hours or days. Forbush decreases can be divided in two classes: sporadic (transient) and recurrent. The sporadic Forbush decreases generate GCR intensity drop lasting 1-2 days then present gradual recovery in, on average, 5-10 days (an example is reported in fig.4; [32]). The sporadic Forbush decreases of the GCR intensity are associated with major solar flares. These Forbush decreases can be caused by (1) shock and ejecta, (2) shock only or (3) ejecta only [33]. Sporadic Forbush decreases generate drops of the order of $5-10 \%$ at $10 G V$ rigidity. The recurrent Forbush effects (with amplitudes $<3-4 \%$ at the rigidity of $10 \mathrm{GV}$ ) are associated with the corotating interaction regions in the interplanetary space. Recurrent Forbush effect has approximately symmetric time-profile: the GCR intensity decreases gradually during 5-7 days and recoveries approximately last the same time.

Forbush effects on the 27-day variation of the GCR intensity are unknown up to present.

Forbush decreases affect high energy GCR more than all other shortterm variations and fluctuations. A detailed study of the LISA-PF test-mass charging variation during a Forbush decrease is in preparation. GCR energy 
differential flux measurements during Forbush decreases were carried out, for example, by the SMILI [34], MASS89 [35] and PAMELA [36] experiments. SMILI and MASS89 are balloon-borne experiments flown from Saskatchwan (Canada) soon before (September 1st) and during (September 5th) the Forbush decrease dated September 4th 1989, respectively. Unfortunately, even if these last two experiments show a low energy modulation of the helium nucleus spectrum due to the Forbush decrease, evident normalization problems between the two experiments do not actually give us the possibility to study accurately the energy dependence of the decrease (see fig.5). This was not the case for the event dated 15 December 2006. The dynamics of the whole event was observed by PAMELA. This satellite experiment, launched on June 15th 2006, is devoted to antimatter search in cosmic rays. Preliminary results were presented by the PAMELA collaboration at the last August European Cosmic-Ray Symposium in Turku (Finland) [37]. In fig.4 the Moscow neutron monitor counting rate represents the trend of the GCR intensity in December 2006. In fig. 6 we have reported the variations of GCR proton flux in different energy intervals (0.4-1 GeV, 1-5 GeV, 5-20 GeV) during the same period as measured by PAMELA. It appears that the Forbush decrease is softened by solar particles below $1 \mathrm{GeV}$ while an intensity drop of up to $30 \%$ is observed between 1 and $5 \mathrm{GeV}$. Some reduction of the flux is found above $10 \mathrm{GeV}$.

\subsection{Hourly and daily GCR fluctuations}

Short-term GCR fluctuations observed near Earth are affected by solarterrestrial relations. However, the balloon-borne BESS-Polar I experiment, flown from Antarctica from December 13th through December 21st 2004 [38], detected galatic proton differential flux variations correlated with the solar activity. Shortly before the BESS-Polar I flight an interplanetary coronal mass ejection or a magnetic cloud reached Earth and on December 14th the count rate of the Bartol South Pole neutron monitor started to recover gradually. The GCR intensity recovery was softened by a high speed stream in the solar wind reaching Earth between December 16th and December 17th. Short-term GCR variations depend on the characteristics of each interplanetary process, however the BESS-Polar I data provide precious clues on these intensity variations as a function of the energy. The proton flux was measured by BESS-Polar I experiment in 4-hour time intervals normalized to the flight average flux in the energy ranges $0.29-0.54 \mathrm{GeV}, 0.54-1 \mathrm{GeV}, 1-3.4$ 


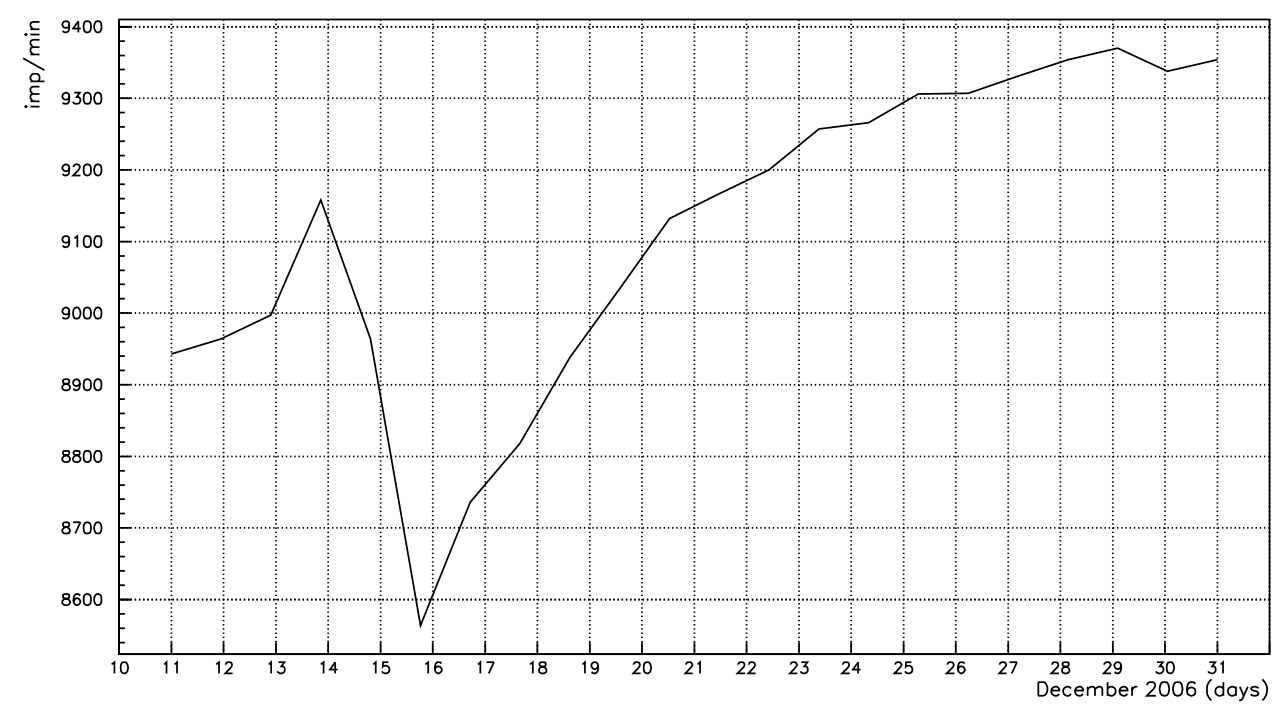

Figure 4: Moscow neutron monitor counting rate in December 2006 [32]. A Forbush decrease occurred on December 15th. We recall that the neutron monitor countrate (Y-axis label) is expressed in neutron impulses per minute. 


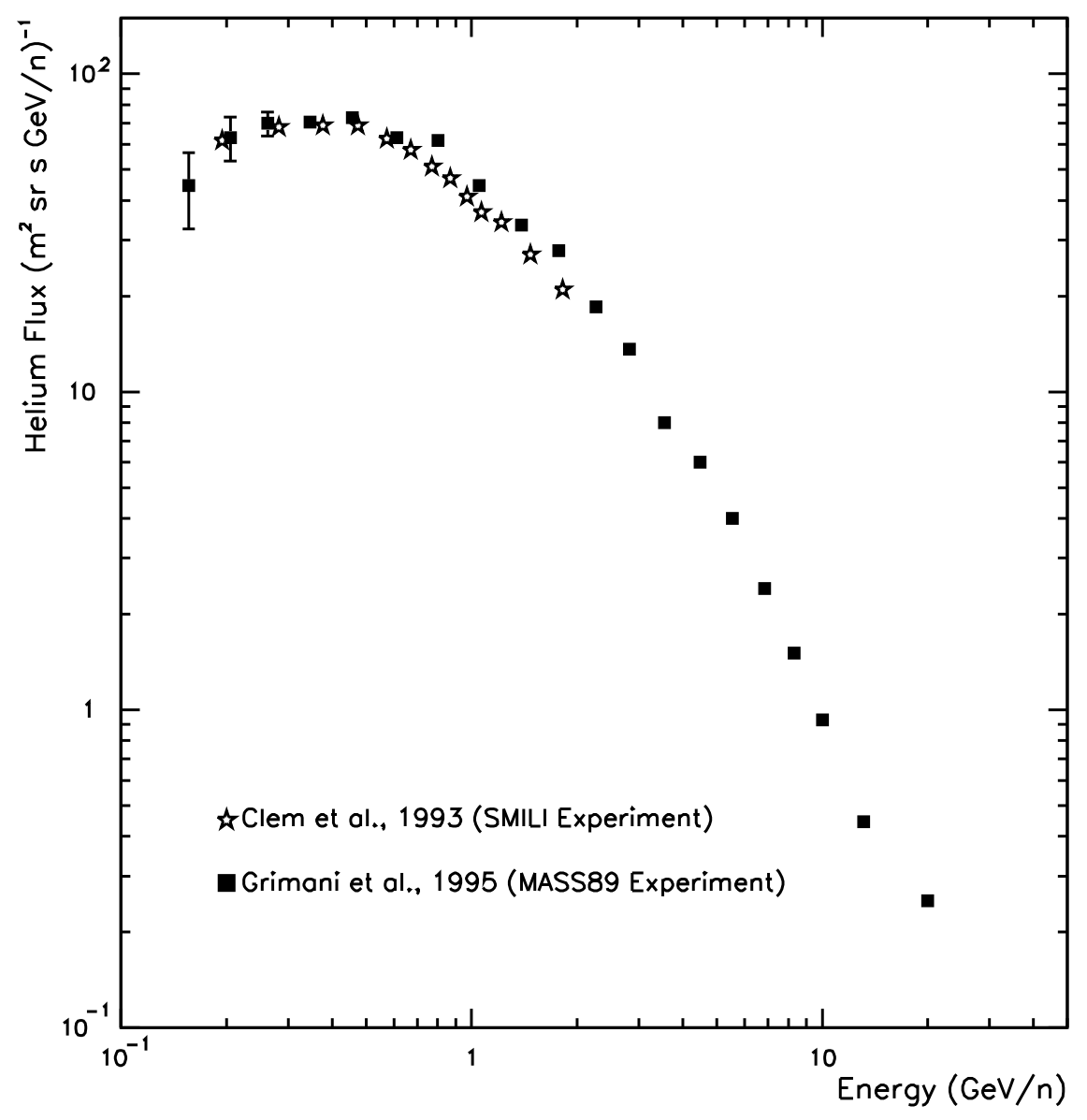

Figure 5: Helium flux measurements carried out by the SMILI and MASS89 experiments on September 1st and 5th 1989, respectively. A Forbush decrease started on September 4th. Normalization problems can be observed between the two experiments since above $1 \mathrm{GeV}$ the SMILI flux assumes smaller values with respect to those of MASS89. 

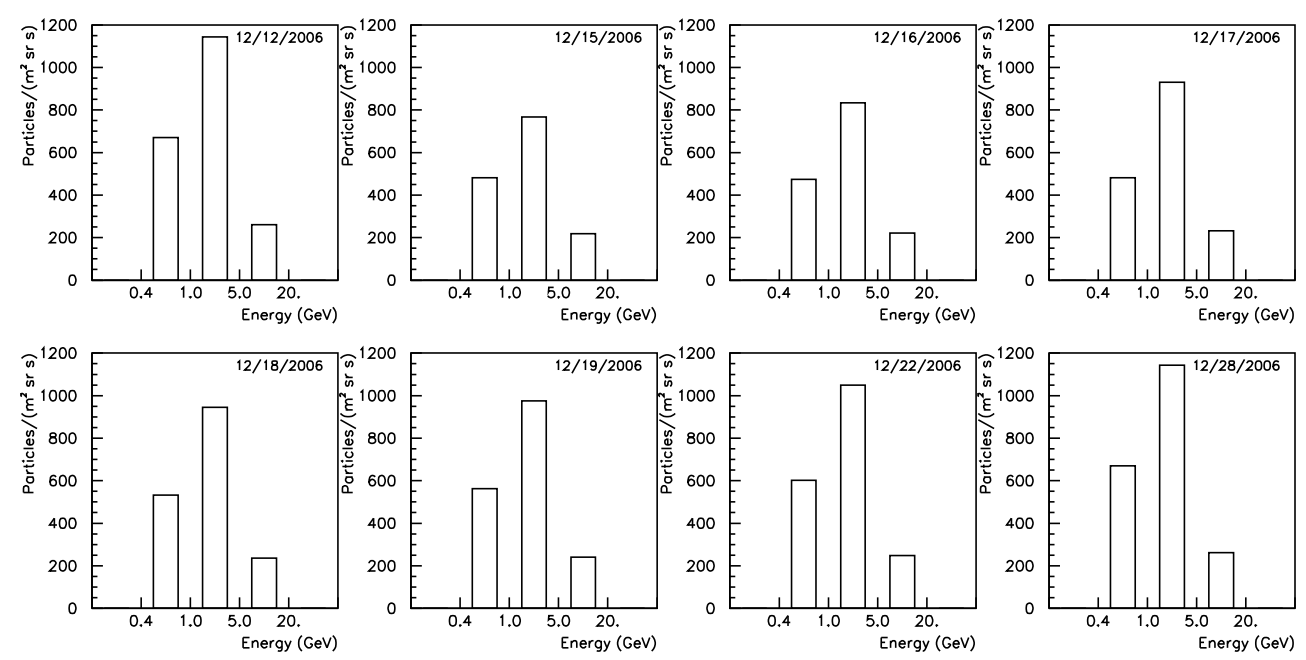

Figure 6: GCR integral proton flux observed by PAMELA in various energy intervals between December 12th and December 28th 2006 [37].

$\mathrm{GeV}$ and 3.4-10 GeV. While diurnal variations do not allow us to give any meaningful explanation to the fluctuations of individual data points [38], a continuous recovery of the low energy differential flux was observed in agreement with the Bartol South Pole neutron monitor trend modulated by the Sun activity. In particular, between $200 \mathrm{MeV}$ and $1 \mathrm{GeV}$ the proton spectrum varies between $-5 \%$ to $+3 \%$ with respect to the average value during the whole flight. Between 1 and $10 \mathrm{GeV}$ the variations appear reduced and consistent with statistical uncertainties [39].

\section{Updated estimate of the number of solar events at the time of LISA-PF}

Nymmik [7]-[8] has found that the SEP fluence distribution follows a powerlaw trend with an exponential decrease for large fluences. This model applies to solar proton fluences ranging between $10^{6}$ and $10^{11}$ protons $\mathrm{cm}^{-2}$ for particle energies above $30 \mathrm{MeV}$. The Nymmik results were inferred from the analysis of the spacecraft IMP-7 and 8 measurements of SEP events during 
the solar cycles 20-22 and from proton fluxes estimated on the basis of radionuclide observations in lunar rocks generated in the last few million years. The Nymmik's model offers the possibility to predict solar events in terms of energy range and of particle peak fluxes instead of fluence only (for a review of other models see [40]-[41] and references therein).

The number of SEP events in individual intervals of fluence during the six months of the expected LISA-PF data taking were estimated according to the March 2010 projections of the number of solar spots reported in fig.1 (see [20] for details).

We have found a minimum, average and maximum number of solar events in 2012 of 2.92, 4.83 and 6.73, respectively. Half of these are expected in six months. The number of events estimated to occur during the LISA-PF mission per interval of fluence appear in fig.7.

\section{Conclusions}

The most recent projections of the solar cycle 24 allow us to estimate the GCR fluxes at the time of future space missions. In addition to long-term variations, short-term fluctuations were considered. In particular, we found that Forbush decreases generate intensity drops up to $30 \%$ in individual energy intervals. Other short-term variations cause maximum variations of a few $\%$ at most. We have also estimated the number of solar events with fluences larger than $10^{6}$ protons $\mathrm{cm}^{-2}$ expected during the second half of 2012 . This number ranges between 1.5 and 3.4, well below previous expectations. This might mean that no events or, at least, no events with fluences equal or larger than $10^{7}$ protons $\mathrm{cm}^{-2}$ might occur at the time of LISA-PF data taking. We point out that $10^{7}$ protons $\mathrm{cm}^{-2}$ is the intensity of events generating a noise larger than the whole LISA budget at low frequencies (at the peak).

\section{Acknowledgements}

CG and MF acknowledge financial support by the Italian Space Agency within the Program "Study exploration of the Solar System". 


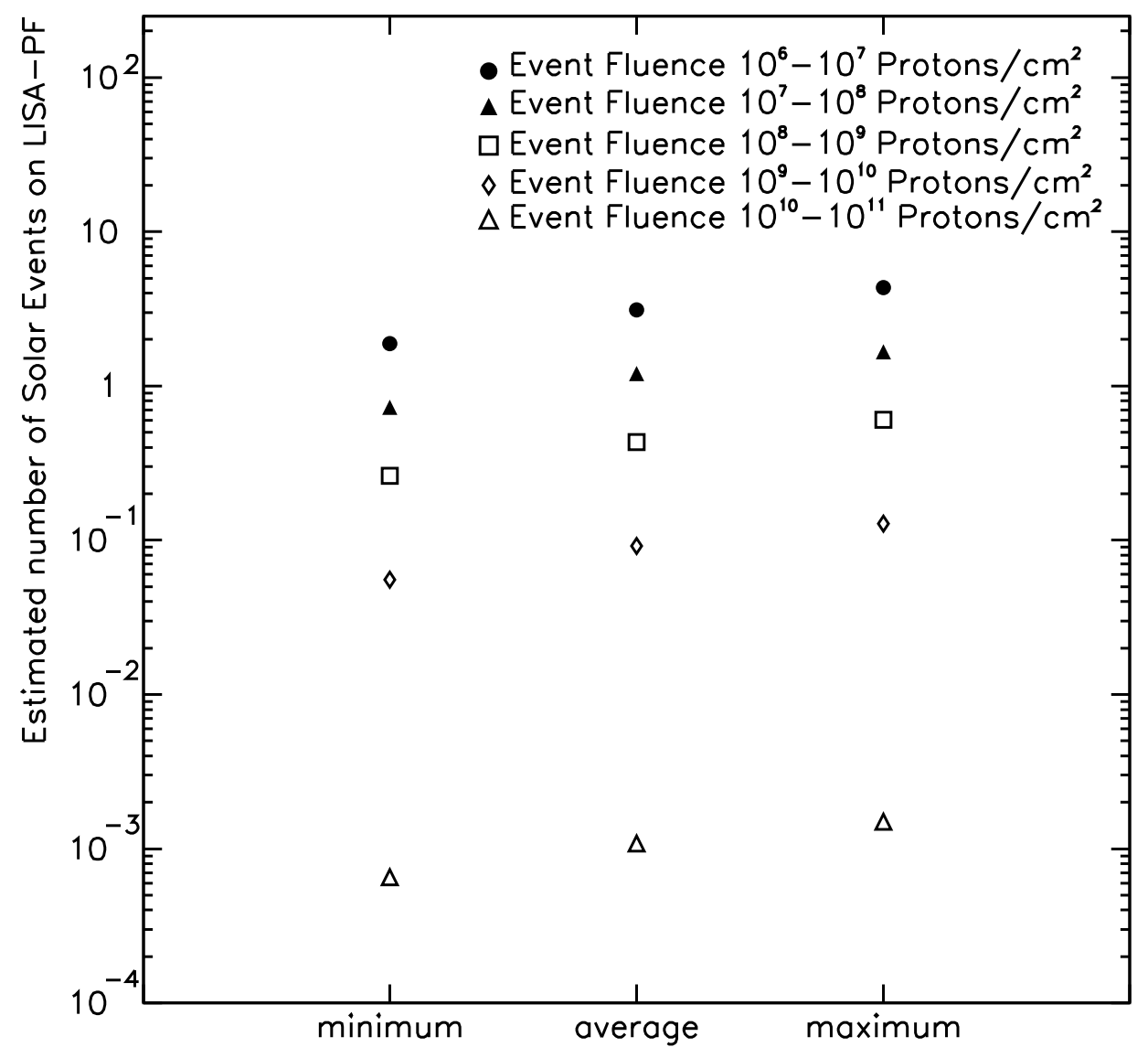

Figure 7: Estimated number of solar events per interval of fluence at the time of LISA-PF data taking. 


\section{References}

[1] Araújo, H. M. et al., 2005, Astropart. Phys., 22, 451, 469

[2] Grimani, C. et al., 2005, Class. Quant. Grav., 22, S327

[3] Shaul, D. N. A. et al., 2006, AIP Conf. Proc., 873, 172

[4] Grimani, C. et al., 2010, J. Phys. Conf. Ser., 228, 012040

[5] Hathaway, D., 2009a, http://solarscience.msfc.nasa.gov/predict. shtml

[6] Hathaway, D., 2009b, http://solarscience.msfc.nasa.gov/images/ssn_ predict.txt

[7] Nymmik, R. A., 1999a, Proc. 26th Int. Cosmic Ray Conf., Salt Lake City, 6, 268

[8] Nymmik, R. A., 1999b, Proc. 26th Int. Cosmic Ray Conf., Salt Lake City, 6, 280

[9] Vitale, S. et al., 2002, Nucl. Phys. B, 110, 209

[10] Hathaway D. and Dikpati M., 2006, http://science.nasa.gov/headlines/ y2006/10may_lagrange.htm

[11] Ferreira, S. E. S. et al., 2003, Ap. J., 594, 552

[12] Bobik P. et al., 2009, WSPC Proc.

[13] Grimani, C. et al., 2007, Proc. 30th Int. Cosmic Ray Conf., Merida

[14] Ting, S., 2010, 22nd Europ. Cosmic Ray Sympos., Turku

[15] Mateos, I. et al., 2010, J. Phys. Conf. Ser., 228, 012039

[16] Starodubtsev, S. A., Usoskin, I. G. and Mursula, K., Solar Physics, 2004, 224, 335

[17] Posnell, W.D., Solar Physics, 2008, 252, 209

[18] Kontor, N. N., 2006, Statistics based regular extrapolation. Prediction submitted 28 August 2006

[19] Li, K.-J., Gao, P.-X., Su, T.-W., 2005, Chin. J. Astron. Astrophys., 5, 539 
[20] Grimani C. et al., 2009a, CQG, 26, 094018

[21] Gleeson, L. J. and Axford, W. I., 1968, Ap. J., 154, 1011

[22] Grimani C. et al., 2009b, Class. Quant. Grav., 26, 215004

[23] Golden, R. L. et al., 1995, Proc. 24th Int. Cosmic Ray Conf., Rome

[24] Wiedenbeck, M. E. et al., Proc. 29th Int. Cosmic Ray Conf., Pune

[25] Alanko-Huotari, K. et al., 2006, Solar Physics, 238, 391

[26] Mitchell, J. W. et al., 2004, Nucl. Phys. B Proc. Suppl., 134, 31

[27] Shikaze, Y. et al., 2007, Astropart. Phys., 28, 154

[28] Boella, G. et al., 2001, J. Geophys. Res., 106, 355

[29] Grimani, C. et al., 2004, Class. Quant. Grav., 21, S629

[30] Gil, A. and Alania, M. V., 2010, Adv. Sp. Res., 45, 429

[31] Alania, M. V., Gil, A. and Modzelewska, R., 2008, Astrophys. Space. Sci. Trans., 4, 31

[32] http://helios.izmiran.troitsk.ru/cosray/main.htm

[33] Kane, R. P., 2010, Ann. Geophys., 28, 479

[34] Clem, J. M., 1993, Proc. 23th Int. Cosmic Ray Conf., Calgary, 3, 707

[35] Grimani, C., 1995, private communication

[36] Casolino, M. et al., 2008, Adv. Sp. Res., 42, 455

[37] Casolino, M. et al., 2010, 22nd Europ. Cosmic Ray Symp. (Turku)

[38] Orito, R. et al., 2009, Proc. 31st Int. Cosmic Ray Conf., Łódź

[39] Hams, T. et al., 2009, Proc. 31st Int. Cosmic Ray Conf., Łódź

[40] Storini, M. et al., 2007, http://cost724.obs.ujf-grenoble.fr/ Documents/chapters/WG1_06_enerpart.pdf

[41] Lantos, P., 2005, Solar Physics, 229, 373 